\title{
Developing Antiepileptogenic Drugs for Acquired Epilepsy: Targeting the Mammalian Target of Rapamycin (mTOR) Pathway
}

\author{
Ling-Hui Zeng, Nicholas R. Rensing, Michael Wong \\ Department of Neurology and the Hope Center for Neurological Disorders, Washington \\ University School of Medicine, St. Louis, Missouri
}

PharmSight on Zeng LH, et al. The mammalian target of rapamycin signaling pathway mediates epileptogenesis in a model of temporal lobe epilepsy. J Neurosci 2009;29:6964-72.

\begin{abstract}
While current medications for epilepsy are primarily symptomatic treatments that suppress seizures, one of the main goals of future drug development in epilepsy is the identification of antiepileptogenic or disease-modifying therapies that can completely prevent epilepsy or slow its progression. A rational antiepileptogenic strategy is to target primary cell signaling pathways that initially trigger the downstream mechanisms causing epileptogenesis. Recent work implicates the mammalian target of rapamycin (mTOR) pathway as mediating epileptogenesis in a genetic epilepsy, Tuberous Sclerosis Complex (TSC), and suggests that mTOR inhibitors, such as rapamycin, may have antiepileptogenic properties for epilepsy in TSC. As mTOR regulates multiple cellular functions that may contribute to epileptogenesis in general, including ion channel expression, synaptic plasticity, and programmed cell death, mTOR inhibitors might also represent an effective antiepileptogenic therapy for other, more common types of epilepsy, such as acquired epilepsies due to brain injuries. Here, we describe evidence from a recently-published study that mTOR mediates epileptogenesis in a popular animal model of acquired limbic epilepsy due to brain injury following kainate-induced status epilepticus, and that rapamycin has antiepileptogenic effects in this model. Furthermore, putative pathways and mechanisms upstream and downstream from mTOR involved in epileptogenesis in the kainate
\end{abstract}

Received 06/15/09; accepted 06/26/09

Correspondence: Michael Wong, M.D., Ph.D., Department of Neurology, Box 8111, Washington University School of Medicine 660 South Euclid Avenue St. Louis, MO 63110, USA. Tel. 314-362-8713, Fax. 314-362-9462. email: wong_m@wustl.edu model are considered, identifying possible additional therapeutic targets. Finally, the potential translational applications of this and other animal model data for developing antiepileptogenic therapies for people with acquired epilepsy due to brain injury are discussed.

Keywords: Epilepsy; Rapamycin; mTOR pathway; Tuberous Sclerosis Complex

Drug development for epilepsy has made substantial progress in recent years. About a dozen new seizure medications have become available for clinical use since the mid-1990's (1). These antiepileptic drugs have helped control seizures and minimize side effects in many patients. Unfortunately, however, these medications have had minimal impact in reducing the number of people with medically-refractory epilepsy, which continues to represent approximately one-third of all epilepsy patients despite multiple trials of both older and newer medications (2). Furthermore, currently available seizure medications are primarily symptomatic treatments that suppress seizures but do not necessarily correct the underlying brain abnormalities causing epilepsy (epileptogenesis) or improve the long-term prognosis of epilepsy (3). Thus, a holy grail of epilepsy drug research has been the pursuit of disease-modifying or "antiepileptogenic" drugs that can slow the progression of epilepsy or completely prevent the development of epilepsy in the first place (4-6).

Many recent antiepileptic drugs were identified through a screening approach, such as via the NIHsponsored Anticonvulsant Drug Development program, in which thousands of compounds were 
tested for efficacy in standard assays of acutelyprovoked seizures in non-epileptic animals. As a result, current seizure medications are effective in suppressing seizures symptomatically and work through mechanisms that directly decrease neuronal excitability, such as modulation of neurotransmitter receptors and ion channels. However, since most of these drugs were not tested in more chronic assays in epileptic animals, it is not surprising that they may not be as effective in preventing the underlying processes of epileptogenesis. Rather than targeting molecular mechanisms that mediate the end-stage symptoms of epilepsy, a more rational strategy for developing antiepileptogenic therapies is to interrupt the early mechanistic processes that initially trigger the cellular and molecular changes in the brain that eventually lead to seizures. In fact, many types of epilepsy are caused by a remote brain injury, such as a stroke or head trauma, in which the patient starts experiencing seizures only after a prolonged period, often spanning months to years, following the brain injury. During this latent period of epileptogenesis, a number of cellular and molecular changes occur in the brain in response to the injury that promote the eventual development of seizures, but also offer a potential window of opportunity for intervening with antiepileptogenic therapies.

While no such antiepileptogenic treatments have yet been established for clinical use, a number of promising avenues have recently started to emerge from basic research. One of the best examples relates to a cell signaling pathway, the mammalian target of rapamycin (mTOR) pathway, which is increasingly recognized to control a variety of important cellular functions under physiological conditions and to contribute to the pathophysiology of a wide range of different diseases (7-9). mTOR is a serine-threonine protein kinase that serves as a central regulator of multiple physiological functions that generally relate to cellular growth, proliferation, metabolism, and survival. A number of upstream pathways, such as phosphatidylinositol-3 kinase (PI3K)/Akt (protein kinase B) and AMP-kinase (AMPK), can activate or inhibit mTOR activity, in response to different extracellular stimuli or intracellular signals, including nutrient and energy status, growth factors, and physiological stress (Figure. 1A). Modulation of mTOR by upstream stimuli and pathways is usually mediated through effects on the tuberous sclerosis complex (TSC) gene products, hamartin and tuberin, and the small GTPase Rheb. In turn, mTOR triggers a number of downstream mechanisms, such as ribosomal S6K/S6 and $4 \mathrm{EBP} 1 / \mathrm{eIF} 4 \mathrm{E}$ pathway, which directly mediate protein synthesis and ribosomal biogenesis related primarily to cellular growth and metabolism (Figure 1A). Thus, under physiological conditions, mTOR is activated by anabolic or energy-rich states, resulting in increased protein synthesis, cell growth, and metabolism. Conversely, in catabolic or energydeficient states, mTOR is inhibited, decreasing protein synthesis and growth. Dysregulation of the mTOR pathway has been implicated in a variety of different diseases, ranging from diabetes and obesity to cardiovascular disease, cancer and neurodegenerative disorders (9). Furthermore, from a translational approach, the clinical availability of mTOR inhibitors, such as rapamycin, conveniently allows the involvement of the mTOR pathway in the pathophysiology of disease to be tested and provides potential novel therapeutic agents for these diseases.

Given the diversity of functions regulated by mTOR, abnormal signaling through the mTOR pathway represents a logical candidate for mediating mechanisms of epileptogenesis. For example, mTOR can affect a variety of cellular and molecular processes, such as neurotransmitter receptor and ion channel expression, synaptic plasticity, neuronal death and apoptosis, and neurogenesis, which influence neuronal excitability and epileptogenesis (10-16). Perhaps the best example of the role of mTOR in epileptogenesis is seen in Tuberous Sclerosis Complex (TSC), which represents one of the most common types of genetic epilepsy. TSC is caused by mutation of one of two genes, the TSC1 and TSC2 genes, and is characterized by tumor or hamartoma formation in multiple organs $(17,18)$. Epilepsy in TSC is very common and usually intractable to available therapies. Although the mechanisms of epileptogenesis are incompletely understood, recent work from human tissue specimens and animal models of TSC has identified a number of cellular and molecular abnormalities in neurons and glia that likely promote epileptogenesis $(19,20)$. As the TSC gene products, hamartin and tuberin, form a complex that normally inhibits the mTOR pathway, TSC gene mutations lead to abnormal hyperactivation of mTOR, which activates multiple downstream mechanisms that mediate many of the phenotypic features of TSC, such as abnormal cell growth and proliferation and tumor formation (Figure 1B). Accordingly, mTOR inhibitors, such as rapamycin, can decrease tumor growth in animal 


\section{A Physiological States}

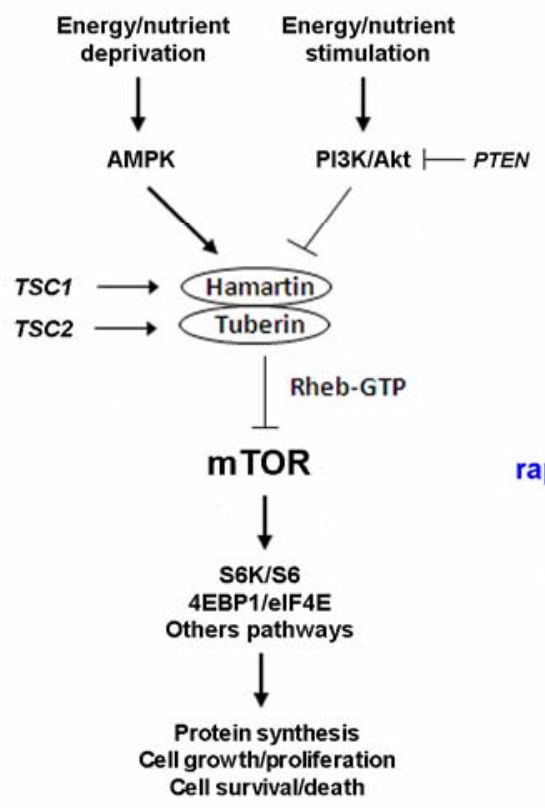

B Tuberous Sclerosis Complex

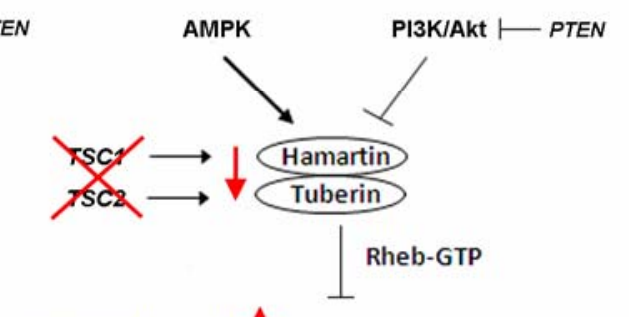

C Acquired Brain Injury

Brain Injury

(TBI, status epilepticus)
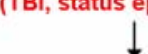

Glutamate Release

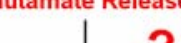

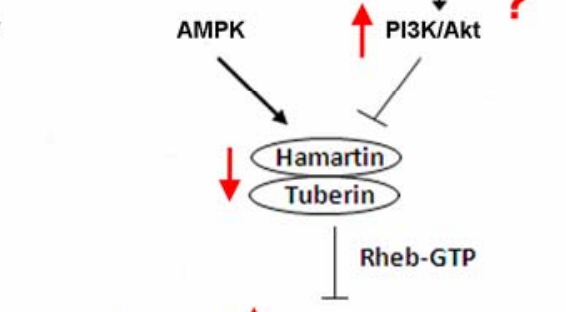

rapamycin $\longrightarrow$ mTOR
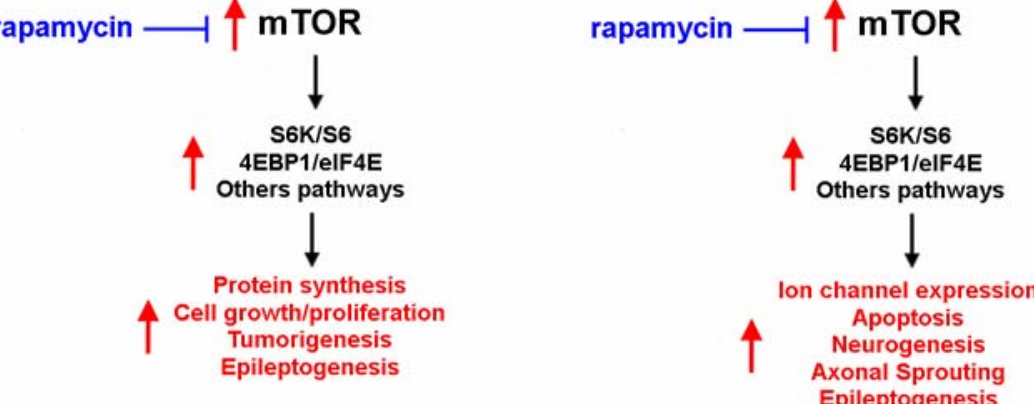

Figure 1. Physiological and pathological regulation of the mTOR pathway. A. Under physiological conditions, mTOR controls multiple downstream effectors, such as S6K/S6 and 4EBP1/elF4E, which regulate protein synthesis and other processes related to cellular growth, proliferation, metabolism, and survival. In response to environmental or physiological stimuli, multiple upstream pathways, primarily involving cascades of protein kinases, may either activate or inhibit mTOR via modulation of the tuberin-hamartin complex and Rheb GTPase. In anabolic states, such as with insulin, growth factors, or nutrient stimulation, some pathways, such as PI3K/Akt, activate the mTOR pathway and induce protein synthesis, cell growth and proliferation. Conversely, in catabolic states, other pathways, such as AMPK, respond to energy/nutrient deprivation by inhibiting the mTOR pathway and thus slowing protein synthesis, cell growth, and metabolism. B. In the disease of TSC, mutation of one of the TSC genes leads to disinhibition or hyperactivation of the mTOR pathway, causing dysregulated protein synthesis, cell growth and proliferation and predisposing to tumorigenesis and epileptogenesis. Mutations in the PTEN gene product, which normally inhibits PI3K/Akt, may lead to similar cellular phenotypes of increased cell growth and proliferation. C. In addition to genetic mutations, acquired brain injuries may cause abnormal activation of the MTOR pathway, which triggers multiple downstream cellular and molecular changes promoting epileptogenesis. Although the specific upstream signaling pathways that are initiated by brain injury to activate mTOR are unknown, a rational mechanism may involve excessive glutamate release, with subsequent activation of the PI3K/Akt pathway. For both TSC and acquired brain injuries, rapamycin may have antiepileptogenic effects by inhibiting mTOR. Note that these schematic figures are oversimplified for clarity, as other upstream regulators, feedback loops, intermediary steps, and alternative pathways are not shown. Abbreviations: 4EBP1 - elongation factor 4E binding protein 1; AMPK - AMP-activated protein kinase; elF4E - elongation initiation factor 4E; Akt - protein kinase B; mTOR mammalian target of rapamycin; PI3K - phosphatidylinositide-3 kinase; PTEN - phosphatase and tensin homolog deleted on chromosome ten; Rheb - Ras homolog expressed in brain; S6 - ribosomal S6; S6K - ribosomal S6 kinase.

models (21), as well as in uncontrolled clinical trials in TSC patients $(22,23)$. Similarly, in mouse models of TSC with abnormal neurological phenotypes, mTOR inhibitors can reverse a number of molecular, histological, and behavioral abnormalities that may relate to epileptogenesis, including megenecephaly, neuronal hypertrophy, and astrogliosis (24-27). Most remarkably, rapamycin treatment, initiated prior to the onset of seizures, prevents the onset of epilepsy in these mice, indicating a potent antiepileptogenic effect (26).

While TSC is a relatively rare genetic disease, there could be significant overlap between mechanisms of epileptogenesis in TSC and other types of epilepsy, such as more common acquired epilepsies due to brain injury. During the latent period between the initial precipitating brain injury and the subsequent onset of epilepsy, different cellular and molecular changes mediating epileptogenesis may occur that involve mTORdependent mechanisms, such as alterations in ion channel expression, aberrant synaptic plasticity, neurogenesis, or apoptosis (Figure 1C). In a recent study, we have begun to test the role of mTOR in a popular animal model of acquired limbic epilepsy due to brain injury. In this model, the glutamate agonist kainate is injected into previously normal mice to induce an acute episode of prolonged 
seizures lasting a few hours (status epilepticus). After recovery from the status epilepticus, the mice enter a latent period of a few days to weeks, during which there are no observable seizures, but a number of cellular changes occur reflecting brain injury and promoting epileptogenesis. The end of the latent period is heralded by the onset of spontaneous seizures and the mice then have epilepsy.

Consistent with a previous report (28), we first demonstrated that the mTOR pathway is abnormally activated by kainate-induced status epilepticus, both acutely during the episode of status epilepticus and more chronically for a few weeks during the latent period of epileptogenesis (29). Rapamycin treatment prevented this inappropriate mTOR activation and also decreased cellular changes that likely contribute to epileptogenesis in this model, including hippocampal neuronal death, neurogenesis, and axonal sprouting. Correspondingly, mTOR inhibition with rapamycin also reduced the development of spontaneous seizures, indicating an antiepileptogenic effect (29). Thus, these studies suggested that mTOR plays a critical role in activating multiple downstream mechanisms of epileptogenesis in the kainate model of acquired limbic epilepsy and that rapamycin has antiepileptogenic actions in this model (Figure 1C).

Although these results are encouraging in extending the pathophysiological importance of mTOR to acquired epilepsies due to brain injury, a number of issues remain unresolved in this regard. First of all, while in TSC there is a direct link between the TSC gene products and mTOR, the biochemical mechanisms by which the mTOR pathway is activated by kainate-induced status epilepticus is unclear. In theory, a number of signaling pathways upstream from mTOR could be triggered by the initial seizures. As large amounts of glutamate are released during status epilepticus, a rational mechanism might start with stimulation of ionotropic or metabolic glutamate receptors and subsequent activation of the PI3K/Akt pathway $(30,31)$, which in turn can increase mTOR activity (Figure 1C). As pharmacological modulators of PI3K and other upstream signaling molecules are available, discovery of the upstream mechanisms that initiate mTOR activation in response to brain injury might lead to additional antiepileptogenic therapies that complement mTOR inhibitors.

Just as the relevant signaling pathways upstream from mTOR remain to be identified, the critical mechanisms of epileptogenesis downstream from mTOR that mediate the antiepileptogenic effects of rapamycin also need to be better defined. Although experiments from the recent study demonstrated that rapamycin prevented putative mechanisms of epileptogenesis in the kainate model, including neuronal death, neurogenesis, and mossy fiber sprouting (29), these findings are only correlative and do not prove that these aberrant cellular processes cause epileptogenesis in this model and are not simply epiphenomena. A number of other downstream mechanisms that are mTORdependent and may contribute to epileptogenesis were not examined in this study, such as ion channel expression, dendritic structure, immune modulation, inflammatory responses, autophagy, and oxidative stress (12-16,32-34). A major issue is whether rapamycin's antiepileptogenic efficacy is primarily related to broader neuroprotective properties in reducing the underlying neuronal death and brain injury, or whether mTOR mediates more specific mechanisms of epileptogenesis independent of cell death. Future studies identifying the most critical downstream mechanisms of epileptogenesis regulated by mTOR may also lead to more specific, targeted antiepileptogenic therapies.

The relevance of mTOR in epileptogenesis in other models and types of both genetic and acquired epilepsy is starting to be explored, further expanding the potential applications and impact of mTOR in epilepsy. Closely related to the kainate model, a recent study indicates that rapamycin can inhibit mossy fiber sprouting in the pilocarpinestatus epilepticus model of acquired limbic epilepsy (35). mTOR activation has also implicated in preliminary experiments of neonatal seizures (36). In mice with inactivation of the PTEN gene, which, similar to the TSC genes, is an upstream inhibitor of mTOR pathway, rapamycin decreases seizures and reverses underlying brain abnormalities $(27,37,38)$. Finally, in models of traumatic brain injury (TBI), mTOR activity is increased and rapamycin exerts neuroprotective effects $(39,40)$. Although the effect of rapamycin on the development of posttraumatic epilepsy has yet not been specifically reported in TBI models, curcumin, which among other properties has recently been found to be an mTOR inhibitor, retards the progression of epileptogenesis in an animal model of TBI $(41,42)$. If rapamycin is also verified to have antiepileptogenic effects in animal models of TBI, this could have direct translational implications, as antiepileptogenic drug trials in TBI patients are very plausible and clinically-relevant $(3,43)$. 
Clinical trials of rapamycin are already in progress studying effects on tumor growth $(22,23)$ and are being planned for epilepsy as a primary endpoint in TSC patients. However, a number of fundamental issues will need to be addressed in the kainate and other animal models before considering an antiepileptogenic drug trial in people with brain injury at risk for epilepsy. First, the existence of a therapeutic window, during which rapamycin will exert antiepileptogenic effects, will need to be defined. For example, what is the longest latency after the initial brain injury (i.e. status epilepticus in the kainate model) at which rapamycin can be initiated in order to still be effective? For how long during the latent period of epileptogenesis does rapamycin need to be administered? Second, even if the efficacy of mTOR inhibitors as antiepileptogenic agents becomes clearly established, more basic science and clinical data are needed about the potential adverse effects of rapamycin, especially in the setting of acute brain injury. Some of the changes in the brain following injury may represent compensatory or adaptive responses promoting recovery from brain injury, such as neurogenesis or synaptic plasticity. Thus, rapamycin could represent a double-edged sword in inhibiting both epileptogenic mechanisms and beneficial, adaptive processes following brain injury. Nevertheless, despite these potential limitations, the recent work with rapamycin is a good sign that drug development for epilepsy is finally shifting from seizure suppression to epilepsy prevention.

\section{Acknowledgements}

Work from the authors has been supported by the National Institutes of Health (K02 NS045583, R01 NS056872), the Tuberous Sclerosis Alliance, and Citizens United for Research in Epilepsy.

\section{Conflicts of Interest}

No potential conflicts of interest to disclose.

\section{References}

1. Beghi E. Efficacy and tolerability of the new antiepileptic drugs: comparison of two recent guidelines. Lancet Neurol 2004;3:618-21.

2. Kwan P, Brodie MJ. Refractory epilepsy: mechanisms and solutions. Expert Rev Neurother 2006;6:397-406.

3. Temkin NR. Antiepileptogenesis and seizure prevention trials with antiepileptic drugs: meta-analysis of controlled trials. Epilepsia 2001;42:515-24.
4. Dichter MA. Models of epileptogenesis in adult animals available for antiepileptogenesis drug screening. Epilepsy Res 2006;68:31-5.

5. Loscher W, Schmidt D. New horizons in the development of antiepileptic drugs: innovative strategies. Epilepsy Res 2006;69:183-272.

6. Stefan H, Lopes da Silva FH, Loscher W. Schmidt D, Perucca E, Brodie MJ, Boon PA, Theodore WH, Moshe SL. Epileptogenesis and rational therapeutic strategies. Acta Neurol Scand 2006;113:139-55.

7. Sarbassov DD, Ali SM, Sabatini DM. Growing roles for the mTOR pathway. Cur Opin Cell Biol 2005;17:596-603.

8. Sandsmark DK, Pelletier C, Weber JD, Gutmann DH. Mammalian target of rapamycin: master regulator of cell growth in the nervous system. Histol Histopathol 2007;22:895-903.

9. Tsang CK, Qi H, Liu LF, Zheng XFS. Targeting mammalian target of rapamycin (mTOR) for health and diseases. Drug Disc Today 2007;12:112-24.

10. Asnaghi L, Bruno P, Priulla M, Nicolin A. mTOR: a protein kinase switching between life and death. Pharmacol Res 2004;50:545-49.

11. Castedo M, Ferri KF, Kroemer G. Mammalian target of rapamycin (mTOR): Pro- and anti-apoptotic. Cell Death Differ 2002;9:99-100.

12. Jaworski J, Spangler S, Seeburg DP, Hoogenraad CC, Sheng M. Control of dendritic arborization by the phosphoinositide-3'kinase-Akt-mammalian target of rapamycin pathway. J Neurosci 2005;25:11300-12.

13. Kumar V, Zhang MX, Swank MW, Kunz J, Wu GY. Regulation of dendritic morphogenesis by Ras-PI3K-AktmTOR and Ras-MAPK signaling pathways. J Neurosci 2005;25:11288-99.

14. Raab-Graham KF, Haddick PC, Jan YN, Jan LY. Activity- and mTOR-dependent suppression of Kv1.1 channel mRNA translation in dendrites. Science 2006;314:144-8.

15. Tang SJ, Reis G, Kang H, Gingras AC, Sonenberg N, Schuman EM. A rapamycin-sensitive signaling pathway contributes to long-term synaptic plasticity in the hippocampus. Proc Natl Acad Sci USA 2002;99:467-72.

16. Wang Y, Barbaro MF, Baraban SC. A role for the mTOR pathway in surface expression of AMPA receptors. Neurosci Lett 2006;401:35-9.

17. Crino PB, Nathanson KL, Henske EP. The tuberous sclerosis complex. N Engl J Med. 2006;355:1345-56.

18. Kwiatkowski DJ. Tuberous Sclerosis: from tubers to mTOR. Ann Hum Genet 2003;67:87-96.

19. Holmes GL, Stafstrom CE, and the Tuberous Sclerosis Study Group. Tuberous Sclerosis Complex and epilepsy: recent developments and future challenges. Epilepsia 2007;48:617-30.

20. Wong M. Mechanisms of epileptogenesis in tuberous sclerosis complex and related malformations of cortical development involving abnormal glioneuronal proliferation. Epilepsia 2008;49:8-21.

21. Lee L, Sudentas P, Donohue B, et al. Efficacy of a rapamycin analog (CCI-779) and IFN-gamma in tuberous 
sclerosis mouse models. Genes Chromosomes Cancer 2005;42:213-27.

22. Franz DN, Leonard J, Tudor C, et al. Rapamycin causes regression of astrocytomas in tuberous sclerosis complex. Ann Neurol 2006;59:490-8.

23. Bissler JJ, McCormack FX, Young LR, et al. Sirolimus for angiomyolipoma in tuberous sclerosis complex or lymphangioleiomyomatosis. N Engl J Med 2008;358:14051.

24. Ehninger D, Han S, Shiyansky C, et al. Reversal of learning deficits in a Tsc2+/- mouse model of tuberous sclerosis. Nat Med 2008;14:843-8.

25. Meikle L, Pollizzi K, Egnor A, et al. Response of a neuronal model of tuberous sclerosis to mammalian target of rapamycin (mTOR) inhibitors: effects on mTORC1 and Akt signaling lead to improved survival and function. J Neurosci 2008;28:5422-32.

26. Zeng LH, Xu L, Gutmann DH, Wong M. Rapamycin prevents epilepsy in a mouse model of tuberous sclerosis complex. Ann Neurol 2008;63:444-53.

27. Zhou J, Blundell J, Ogawa S, et al. Pharmacological inhibition of mTORC1 suppresses anatomical, cellular, and behavioral abnormalities in neural-specific Pten knock-out mice. J Neurosci 2009;29:1773-83.

28. Shacka JJ, Lu J, Xie ZL, Uchiyama Y, Roth KA, Zhang J. Kainic acid induces early and transient autophagic stress in mouse hippocampus. Neurosci Lett 2007;414:5760.

29. Zeng LH, Rensing NR, Wong M. The mammalian target of rapamycin signaling pathway mediates epileptogenesis in a model of temporal lobe epilepsy. J Neurosci 2009;29:6964-72.

30. Sutton G, Chandler LJ. Activity-dependent NMDA receptor-mediated activation of protein kinase $\mathrm{B} / \mathrm{Akt}$ in cortical neuronal cultures. J Neurochem 2002;82:10971105.

31. Hou L, Klann E. Activation of the phosphoinositide 3kinase-Akt-mammalian target of rapamycin signaling pathway is required for metabotropic glutamate receptordependent long-term depression. J Neurosci 2004;24:6352-61.

32. Pan T, Kondo S, Zhu W, Xie W, Jankovic J, Le W. Neuroprotection of rapamycin in lactacystin-induced neurodegeneration via autophagy enhancement. Neurobiol Dis 2008;32:16-25.

33. Di Nardo A, Kramvis I, Cho N, et al. Tuberous sclerosis complex activity is required to control neuronal stress responses in an mTOR-dependent manner. J Neurosci 2009;29:5926-37.

34. Thomson AW, Turnquist HR, Raimondi G. Immunoregulatory functions of mTOR inhibition. Nature Rev Immuno 2009;9:324-37.

35. Buckmaster PS, Ingram E, Wen X. Inhibition of the mammalian target of rapamycin signaling pathway suppresses dentate granule cell axon sprouting in a rodent model of temporal lobe epilepsy. J Neurosci 2009;29:825969.

36. Zhou X, Lan VJ, Fitzgerald E, Talos DM, Jensen FE. Seizure-induced upregulation of the mammalian target of rapamycin (mTOR) signaling pathway in the developing non-TSC rat brain. Epilepsia 2008;49(Suppl7):325.

37. Kwon $\mathrm{CH}$, Zhu X, Zhang J, Baker SJ. mTOR is required for hypertrophy of Pten-deficient neuronal soma in vivo. Proc Natl Acad Sci USA 2003;100:12923-8.

38. Ljungberg MC, Sunnen CN, Lugo JN, Anderson AE, D'Arcangelo G. Rapamycin suppresses seizures and neuronal hypertrophy in a mouse model of cortical dysplasia. Dis Model Mech 2009;2:389-98.

39. Chen S, Atkins CM, Liu CL, Alonso OF, Dietrich WD, $\mathrm{Hu} \mathrm{BR}$. Alterations in mammalian target of rapamycin signaling pathways after traumatic brain injury. J Cereb Blood Flow Metab 2007;27:939-49.

40. Erlich S, Alexandrovich A, Shohami E, PinkasKramarski R. Rapamycin is a neuroprotective treatment for traumatic brain injury. Neurobiol Dis 2007;26:86-93.

41. Beevers CS, Chen L, Liu L, Luo Y, Webster NJG, Huang S. Curcumin disrupts the mammalian target of rapamycin-raptor complex. Cancer Res 2009;69:1000-8.

42. Jyoti A, Sethi P, Sharma D. Curcumin protects against electrobehavioral progression of seizures in the ironinduced experimental model of epileptogenesis. Epilepsy Behav 2009;14:300-8.

43. Temkin NR, Dikmen SS, Wilensky AJ, Keihm J, Chabal S, Winn HR. A randomized, double-blind study of phenytoin for the prevention of post-traumatic seizures. $\mathrm{N}$ Engl J Med 1990;323:497-502. 